Acta Crystallographica Section E

Structure Reports

Online

ISSN 1600-5368

\section{2-Methylsulfonyl-1,2,4-triazolo[1,5-a]- quinazolin-5(4H)-one}

\section{Rashad Al-Salahi, ${ }^{a}$ Mohamed Marzouk,, Mohammed Abbas ${ }^{a}$ and Seik Weng $\mathrm{Ng}^{\mathrm{b}, \mathrm{c} *}$}

a Department of Pharmaceutical Chemistry, College of Pharmacy, King Saud University, Riyadh 11451, Saudi Arabia, ${ }^{\mathbf{b}}$ Department of Chemistry, University of Malaya, 50603 Kuala Lumpur, Malaysia, and ${ }^{\mathbf{C}}$ Chemistry Department, Faculty of Science, King Abdulaziz University, PO Box 80203 Jeddah, Saudi Arabia

Correspondence e-mail: seikweng@um.edu.my

Received 10 May 2012; accepted 14 May 2012

Key indicators: single-crystal X-ray study; $T=294 \mathrm{~K}$; mean $\sigma(\mathrm{C}-\mathrm{C})=0.003 \AA$; $R$ factor $=0.030 ; w R$ factor $=0.082 ;$ data-to-parameter ratio $=13.7$.

The triazoloquinazoline fused-ring system of the title compound, $\mathrm{C}_{10} \mathrm{H}_{8} \mathrm{~N}_{4} \mathrm{O}_{3} \mathrm{~S}$, is essentially planar (r.m.s. deviation $=0.027 \AA$ ). In the crystal, adjacent molecules are linked by $\mathrm{N}-\mathrm{H} \cdots \mathrm{O}_{\text {sulfonyl }}$ hydrogen bonds, generating a helical chain running along the $b$ axis.

\section{Related literature}

For the synthesis of the precursor, see: Al-Salahi \& Geffken (2011).

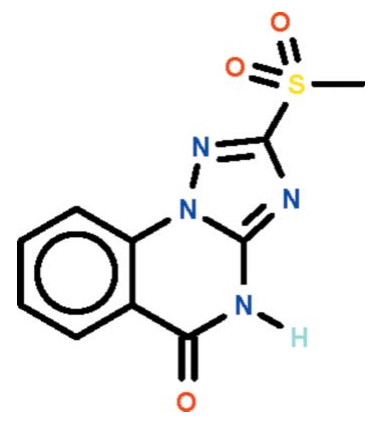

\section{Experimental}

\section{Crystal data}

\section{$\mathrm{C}_{10} \mathrm{H}_{8} \mathrm{~N}_{4} \mathrm{O}_{3} \mathrm{~S}$}

$M_{r}=264.26$

Monoclinic, $P 2_{1}$ $\beta=106.628(2)^{\circ}$

$V=551.73(2) \AA^{3}$

$Z=2$

$\mathrm{Cu} K \alpha$ radiation

Data collection

Agilent SuperNova Dual diffractometer with an Atlas detector

Absorption correction: multi-scan (CrysAlis PRO; Agilent, 2012)

$T_{\min }=0.613, T_{\max }=0.948$

Refinement

$R\left[F^{2}>2 \sigma\left(F^{2}\right)\right]=0.030$

$w R\left(F^{2}\right)=0.082$

$S=1.04$

2304 reflections

168 parameters

1 restraint $\mu=2.71 \mathrm{~mm}^{-1}$

$T=294 \mathrm{~K}$

$0.20 \times 0.10 \times 0.02 \mathrm{~mm}$

9640 measured reflections 2304 independent reflections 2237 reflections with $I>2 \sigma(I)$ $R_{\text {int }}=0.025$

Table 1

Hydrogen-bond geometry $\left(\AA{ }^{\circ}\right)$.

\begin{tabular}{lllll}
\hline$D-\mathrm{H} \cdots A$ & $D-\mathrm{H}$ & $\mathrm{H} \cdots A$ & $D \cdots A$ & $D-\mathrm{H} \cdots A$ \\
\hline $\mathrm{N} 1-\mathrm{H} 1 \cdots \mathrm{O} 2{ }^{\mathrm{i}}$ & $0.88(1)$ & $2.23(1)$ & $3.072(2)$ & $160(3)$ \\
\hline
\end{tabular}

Symmetry code: (i) $-x+1, y+\frac{1}{2},-z$.

Data collection: CrysAlis PRO (Agilent, 2012); cell refinement: CrysAlis PRO; data reduction: CrysAlis PRO; program(s) used to solve structure: SHELXS97 (Sheldrick, 2008); program(s) used to refine structure: SHELXL97 (Sheldrick, 2008); molecular graphics: $X$-SEED (Barbour, 2001); software used to prepare material for publication: publCIF (Westrip, 2010).

We thank the Research Center of the College of Pharmacy College and Deanship of Scientific Research of King Saud University, and the Ministry of Higher Education of Malaysia (grant No. UM.C/HIR/MOHE/SC/12) for supporting this study.

Supplementary data and figures for this paper are available from the IUCr electronic archives (Reference: BT5915).

\title{
References
}

Agilent (2012). Crys Alis PRO. Agilent Technologies, Yarnton, England. Al-Salahi, R. \& Geffken, D. (2011). Synth. Commun. 41, 3512-3523. Barbour, L. J. (2001). J. Supramol. Chem. 1, 189-191.

Flack, H. D. (1983). Acta Cryst. A39, 876-881.

Sheldrick, G. M. (2008). Acta Cryst. A64, 112-122.

Westrip, S. P. (2010). J. Appl. Cryst. 43, 920-925. 


\section{supporting information}

Acta Cryst. (2012). E68, o1806 [doi:10.1107/S1600536812021769]

\section{2-Methylsulfonyl-1,2,4-triazolo[1,5-a]quinazolin-5(4H)-one}

\section{Rashad Al-Salahi, Mohamed Marzouk, Mohammed Abbas and Seik Weng Ng}

\section{S1. Comment}

2-(Methylsulfanyl)-[1,2,4]triazolo[1,5-a]quinazolin-5-one was synthesized from 2-hydrazinobenzoic acid and dimethyl $\mathrm{N}$-cyanoimidodithiocarbonate; further reactions on the inherent lactam unit yielded other derivatives (Al-Salahi \& Geffken, 2011). In the present study, this compound is oxided by hydrogen peroxide. The triazoloquinazoline fused-ring system of $\mathrm{C}_{10} \mathrm{H}_{8} \mathrm{~N}_{4} \mathrm{O}_{3} \mathrm{~S}$ (Scheme I, Fig. 1) is planar. Adjacent molecules are linked by an $N-H \cdots \mathrm{O}_{\text {sulfonyl }}$ hydrogen bond to generate a helical chain running along the $b$-axis of the monoclinic unit cell (Fig. 2, Table 1).

\section{S2. Experimental}

Under ice-cold conditions, 2-hydrazinobenzoic acid (10 mmol, $1.52 \mathrm{~g}$ ) was added to a solution of dimethyl $\mathrm{N}$-cyanodithioimidocarbonate $(10 \mathrm{mmol}, 1.46 \mathrm{~g})$ in ethanol $(20 \mathrm{ml})$. Triethylamine $(30 \mathrm{mmol}, 3.03 \mathrm{~g})$ was added. The reaction mixture was stirred overnight at room temperature. Concentrated hydrochloric acid was added; the acidified mixture for heated for an hour. The mixture was poured into ice water; the solid that formed was collected and recrystallized from ethanol to give colorless crystals of 2-(methylsulfanyl)-[1,2,4]triazolo[1,5- $a]$ quinazolin-5-one. The procedure was that reported earlier (Al-Salahi \& Geffken, 2011).

To the boiling mixture of 2-methylsulfanyl-[1,2,4]triazolo[1,5-a]quinazolin-5-one (1 mmol, $0.23 \mathrm{~g})$ in glacial acetic acid $(5 \mathrm{ml})$ was added hydrogen peroxide. Colorless crystals of the oxidized product were obtained when the solution was allowed to cool.

\section{S3. Refinement}

All $\mathrm{H}$-atom were located in a difference Fourier map. Carbon-bound $\mathrm{H}$-atoms were placed in calculated positions [C-H 0.93 to $\left.0.96 \AA, U_{\text {iso }}(\mathrm{H}) 1.2-1.5 U_{\text {eq }}(\mathrm{C})\right]$ and were included in the refinement in the riding model approximation.

The amino $\mathrm{H}$-atom was refined isotropically with a distance restraint of $\mathrm{N}-\mathrm{H} 0.88 \pm 0.01 \AA$. 


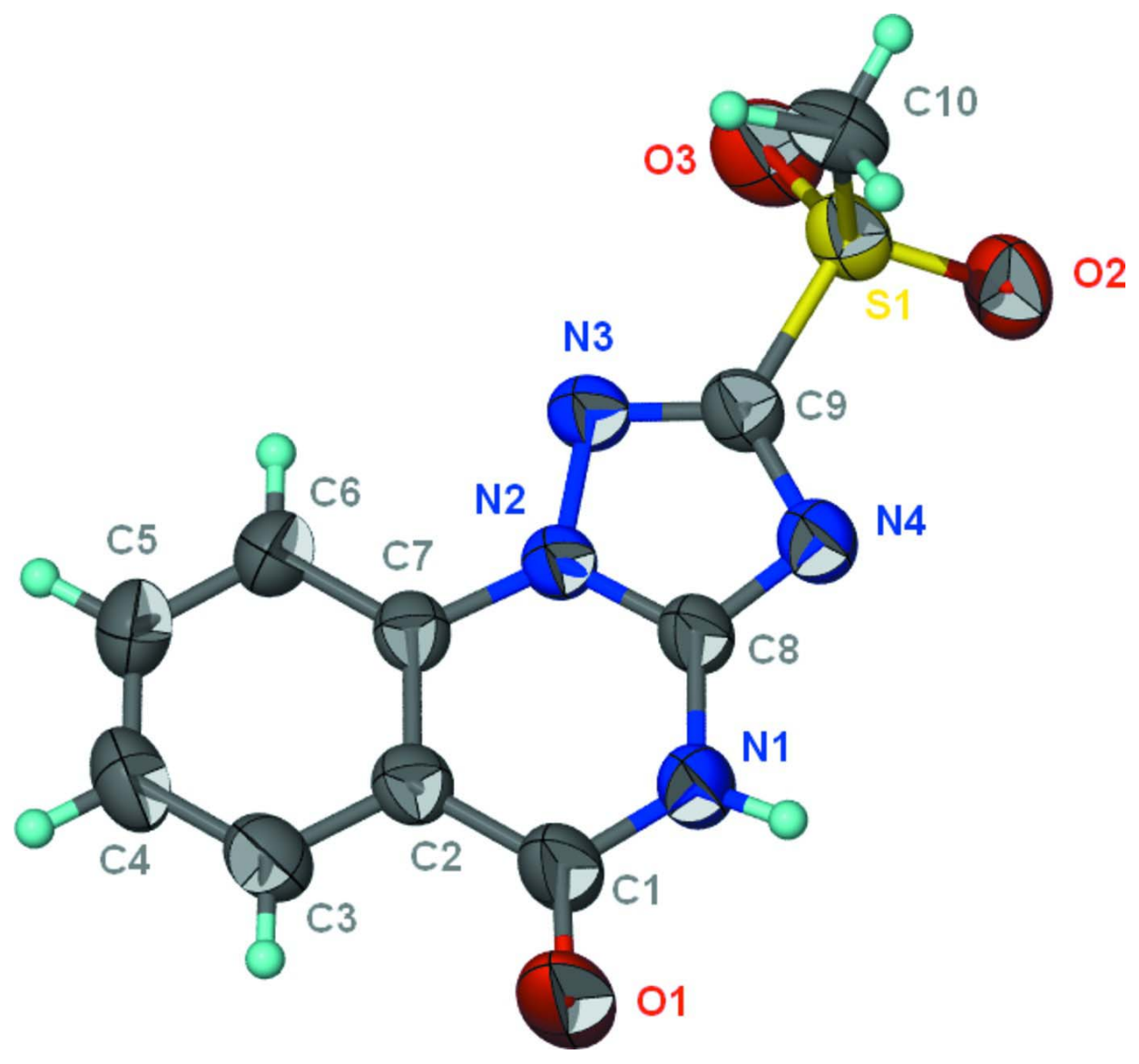

Figure 1

Anisotropic displacement ellipsoid plot (Barbour, 2001) of $\mathrm{C}_{10} \mathrm{H}_{8} \mathrm{~N}_{4} \mathrm{O}_{3} \mathrm{~S}$ at the $70 \%$ probability level; hydrogen atoms are drawn as spheres of arbitrary radius. 


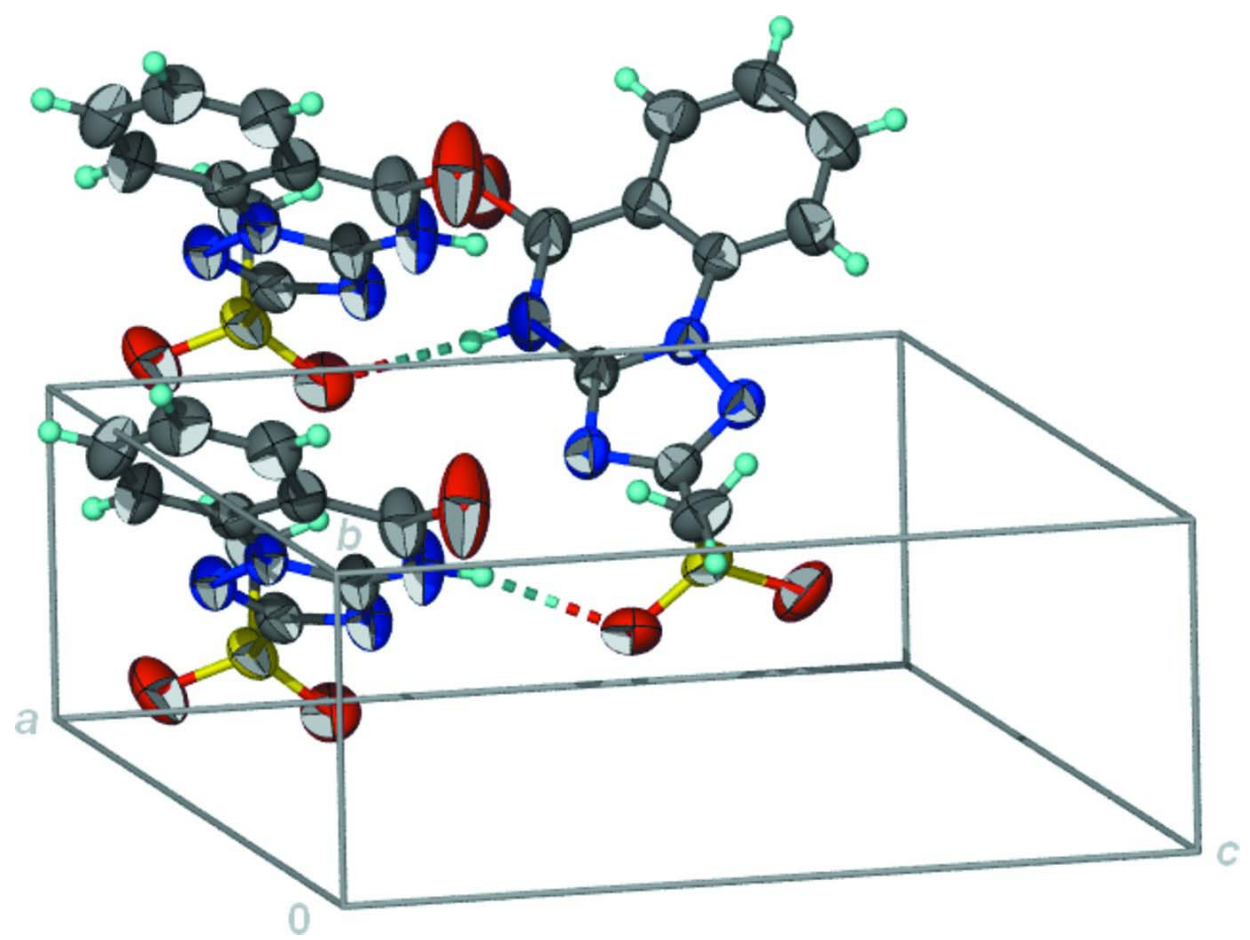

Figure 2

Hydrogen-bonded chain motif.

\section{2-Methylsulfonyl-1,2,4-triazolo[1,5-a]quinazolin-5(4H)-one}

\section{Crystal data}

$\mathrm{C}_{10} \mathrm{H}_{8} \mathrm{~N}_{4} \mathrm{O}_{3} \mathrm{~S}$

$M_{r}=264.26$

Monoclinic, $P 2_{1}$

Hall symbol: $\mathrm{P} 2 \mathrm{yb}$

$a=9.6216(2) \AA$

$b=4.9206(1) \AA$

$c=12.1623(3) \AA$

$\beta=106.628(2)^{\circ}$

$V=551.73(2) \AA^{3}$

$Z=2$

\section{Data collection}

Agilent SuperNova Dual

diffractometer with an Atlas detector

Radiation source: SuperNova $(\mathrm{Cu}) \mathrm{X}$-ray

Source

Mirror monochromator

Detector resolution: 10.4041 pixels $\mathrm{mm}^{-1}$

$\omega$ scan

Absorption correction: multi-scan

(CrysAlis PRO; Agilent, 2012)
$F(000)=272$

$D_{\mathrm{x}}=1.591 \mathrm{Mg} \mathrm{m}^{-3}$

$\mathrm{Cu} K \alpha$ radiation, $\lambda=1.54184 \AA$

Cell parameters from 6133 reflections

$\theta=3.8-76.5^{\circ}$

$\mu=2.71 \mathrm{~mm}^{-1}$

$T=294 \mathrm{~K}$

Plate, colorless

$0.20 \times 0.10 \times 0.02 \mathrm{~mm}$

$T_{\min }=0.613, T_{\max }=0.948$

9640 measured reflections

2304 independent reflections

2237 reflections with $I>2 \sigma(I)$

$R_{\text {int }}=0.025$

$\theta_{\text {max }}=76.7^{\circ}, \theta_{\text {min }}=3.8^{\circ}$

$h=-12 \rightarrow 12$

$k=-6 \rightarrow 6$

$l=-14 \rightarrow 15$ 


\section{Refinement}

Refinement on $F^{2}$

Least-squares matrix: full

$R\left[F^{2}>2 \sigma\left(F^{2}\right)\right]=0.030$

$w R\left(F^{2}\right)=0.082$

$S=1.04$

2304 reflections

168 parameters

1 restraint

Primary atom site location: structure-invariant direct methods

Secondary atom site location: difference Fourier map
Hydrogen site location: inferred from neighbouring sites

$\mathrm{H}$ atoms treated by a mixture of independent and constrained refinement

$w=1 /\left[\sigma^{2}\left(F_{\mathrm{o}}^{2}\right)+(0.0569 P)^{2}+0.056 P\right]$ where $P=\left(F_{\mathrm{o}}^{2}+2 F_{\mathrm{c}}^{2}\right) / 3$

$(\Delta / \sigma)_{\max }=0.001$

$\Delta \rho_{\max }=0.15$ e $\AA^{-3}$

$\Delta \rho_{\min }=-0.26$ e $\AA^{-3}$

Absolute structure: Flack (1983), 1007 Friedel pairs

Absolute structure parameter: $0.00(2)$

Fractional atomic coordinates and isotropic or equivalent isotropic displacement parameters $\left(\AA^{2}\right)$

\begin{tabular}{lllll}
\hline & $x$ & $y$ & $z$ & $U_{\text {iso }} * / U_{\text {eq }}$ \\
\hline S1 & $0.24294(4)$ & $0.00109(9)$ & $0.18198(3)$ & $0.04202(13)$ \\
O1 & $0.8628(2)$ & $0.7880(6)$ & $0.13678(16)$ & $0.0903(8)$ \\
O2 & $0.24690(17)$ & $-0.1892(3)$ & $0.09276(13)$ & $0.0566(4)$ \\
O3 & $0.23731(18)$ & $-0.0963(4)$ & $0.29138(14)$ & $0.0645(4)$ \\
N1 & $0.67906(18)$ & $0.4984(5)$ & $0.12477(13)$ & $0.0542(4)$ \\
H1 & $0.677(3)$ & $0.451(7)$ & $0.0548(13)$ & $0.086(9)^{*}$ \\
N2 & $0.56362(13)$ & $0.4813(4)$ & $0.27059(11)$ & $0.0354(3)$ \\
N3 & $0.45055(15)$ & $0.3499(3)$ & $0.29552(13)$ & $0.0392(3)$ \\
N4 & $0.47068(16)$ & $0.2190(4)$ & $0.12045(12)$ & $0.0436(4)$ \\
C1 & $0.7743(2)$ & $0.6953(5)$ & $0.18009(17)$ & $0.0528(5)$ \\
C2 & $0.75983(17)$ & $0.7852(4)$ & $0.29235(15)$ & $0.0396(4)$ \\
C3 & $0.85191(19)$ & $0.9855(5)$ & $0.35516(16)$ & $0.0478(4)$ \\
H3 & 0.9222 & 1.0634 & 0.3262 & $0.057^{*}$ \\
C4 & $0.8387(2)$ & $1.0679(4)$ & $0.45993(18)$ & $0.0536(5)$ \\
H4 & 0.8999 & 1.2021 & 0.5012 & $0.064^{*}$ \\
C5 & $0.7349(2)$ & $0.9521(5)$ & $0.50437(17)$ & $0.0534(5)$ \\
H5 & 0.7273 & 1.0095 & 0.5753 & $0.064^{*}$ \\
C6 & $0.6429(2)$ & $0.7530(5)$ & $0.44484(16)$ & $0.0472(4)$ \\
H6 & 0.5743 & 0.6733 & 0.4752 & $0.057^{*}$ \\
C7 & $0.65534(17)$ & $0.6748(3)$ & $0.33865(14)$ & $0.0356(3)$ \\
C8 & $0.57227(18)$ & $0.3991(4)$ & $0.16704(14)$ & $0.0389(4)$ \\
C9 & $0.40156(18)$ & $0.2013(4)$ & $0.20289(14)$ & $0.0382(4)$ \\
C10 & $0.1035(2)$ & $0.2349(4)$ & $0.12929(18)$ & $0.0500(5)$ \\
H10A & 0.0116 & 0.1442 & 0.1142 & $0.075^{*}$ \\
H10B & 0.1129 & 0.3136 & 0.0596 & $0.075^{*}$ \\
H10C & 0.1091 & 0.3755 & 0.1851 & $0.075^{*}$ \\
& & & & \\
& & & &
\end{tabular}

Atomic displacement parameters $\left(\AA^{2}\right)$

\begin{tabular}{lllllll}
\hline & $U^{11}$ & $U^{22}$ & $U^{33}$ & $U^{12}$ & $U^{13}$ & $U^{23}$ \\
\hline S1 & $0.0482(2)$ & $0.0365(2)$ & $0.0405(2)$ & $-0.01197(18)$ & $0.01137(15)$ & $0.00488(17)$ \\
O1 & $0.0929(13)$ & $0.131(2)$ & $0.0609(10)$ & $-0.0698(14)$ & $0.0448(10)$ & $-0.0294(12)$
\end{tabular}


supporting information

$\begin{array}{lllllll}\text { O2 } & 0.0684(9) & 0.0428(8) & 0.0593(9) & -0.0175(7) & 0.0197(7) & -0.0074(7) \\ \text { O3 } & 0.0741(10) & 0.0654(10) & 0.0541(8) & -0.0176(8) & 0.0183(7) & 0.0194(7) \\ \text { N1 } & 0.0573(9) & 0.0762(11) & 0.0343(7) & -0.0280(10) & 0.0216(6) & -0.0137(9) \\ \text { N2 } & 0.0342(6) & 0.0410(7) & 0.0312(6) & -0.0058(6) & 0.0100(5) & -0.0011(6) \\ \text { N3 } & 0.0387(7) & 0.0435(8) & 0.0372(7) & -0.0075(6) & 0.0138(6) & -0.0003(6) \\ \text { N4 } & 0.0456(8) & 0.0505(9) & 0.0342(7) & -0.0125(7) & 0.0107(6) & -0.0036(6) \\ \text { C1 } & 0.0521(10) & 0.0692(14) & 0.0385(9) & -0.0229(10) & 0.0150(8) & -0.0051(9) \\ \text { C2 } & 0.0359(8) & 0.0446(9) & 0.0360(8) & -0.0031(7) & 0.0064(6) & 0.0006(7) \\ \text { C3 } & 0.0431(8) & 0.0498(10) & 0.0471(9) & -0.0101(9) & 0.0073(7) & -0.0003(10) \\ \text { C4 } & 0.0515(11) & 0.0499(13) & 0.0516(11) & -0.0093(8) & 0.0022(9) & -0.0136(8) \\ \text { C5 } & 0.0555(10) & 0.0585(15) & 0.0452(9) & -0.0048(10) & 0.0126(8) & -0.0181(9) \\ \text { C6 } & 0.0476(9) & 0.0554(12) & 0.0412(9) & -0.0052(9) & 0.0169(7) & -0.0098(9) \\ \text { C7 } & 0.0342(7) & 0.0369(8) & 0.0332(7) & 0.0004(6) & 0.0055(6) & -0.0014(6) \\ \text { C8 } & 0.0389(8) & 0.0471(9) & 0.0296(8) & -0.0095(7) & 0.0079(6) & -0.0017(7) \\ \text { C9 } & 0.0392(8) & 0.0380(8) & 0.0360(8) & -0.0059(7) & 0.0084(6) & 0.0027(7) \\ \text { C10 } & 0.0407(9) & 0.0498(11) & 0.0585(11) & -0.0090(8) & 0.0127(8) & 0.0067(9) \\ \end{array}$

Geometric parameters $\left(\AA,{ }^{\circ}\right)$

\begin{tabular}{|c|c|c|c|}
\hline $\mathrm{S} 1-\mathrm{O} 3$ & $1.4296(16)$ & $\mathrm{C} 1-\mathrm{C} 2$ & $1.479(3)$ \\
\hline $\mathrm{S} 1-\mathrm{O} 2$ & $1.4421(16)$ & $\mathrm{C} 2-\mathrm{C} 7$ & $1.395(2)$ \\
\hline $\mathrm{S} 1-\mathrm{C} 10$ & $1.744(2)$ & $\mathrm{C} 2-\mathrm{C} 3$ & $1.397(3)$ \\
\hline $\mathrm{S} 1-\mathrm{C} 9$ & $1.7726(17)$ & $\mathrm{C} 3-\mathrm{C} 4$ & $1.377(3)$ \\
\hline $\mathrm{O} 1-\mathrm{C} 1$ & $1.211(2)$ & $\mathrm{C} 3-\mathrm{H} 3$ & 0.9300 \\
\hline $\mathrm{N} 1-\mathrm{C} 8$ & $1.364(2)$ & $\mathrm{C} 4-\mathrm{C} 5$ & $1.387(3)$ \\
\hline $\mathrm{N} 1-\mathrm{C} 1$ & $1.370(3)$ & $\mathrm{C} 4-\mathrm{H} 4$ & 0.9300 \\
\hline $\mathrm{N} 1-\mathrm{H} 1$ & $0.877(10)$ & $\mathrm{C} 5-\mathrm{C} 6$ & $1.379(3)$ \\
\hline $\mathrm{N} 2-\mathrm{C} 8$ & $1.348(2)$ & $\mathrm{C} 5-\mathrm{H} 5$ & 0.9300 \\
\hline $\mathrm{N} 2-\mathrm{N} 3$ & $1.3718(19)$ & $\mathrm{C} 6-\mathrm{C} 7$ & $1.385(2)$ \\
\hline $\mathrm{N} 2-\mathrm{C} 7$ & $1.397(2)$ & $\mathrm{C} 6-\mathrm{H} 6$ & 0.9300 \\
\hline $\mathrm{N} 3-\mathrm{C} 9$ & $1.312(2)$ & $\mathrm{C} 10-\mathrm{H} 10 \mathrm{~A}$ & 0.9600 \\
\hline $\mathrm{N} 4-\mathrm{C} 8$ & $1.321(2)$ & $\mathrm{C} 10-\mathrm{H} 10 \mathrm{~B}$ & 0.9600 \\
\hline $\mathrm{N} 4-\mathrm{C} 9$ & $1.355(2)$ & $\mathrm{C} 10-\mathrm{H} 10 \mathrm{C}$ & 0.9600 \\
\hline $\mathrm{O} 3-\mathrm{S} 1-\mathrm{O} 2$ & $119.93(11)$ & $\mathrm{C} 3-\mathrm{C} 4-\mathrm{H} 4$ & 119.8 \\
\hline $\mathrm{O} 3-\mathrm{S} 1-\mathrm{C} 10$ & 109.44 (11) & $\mathrm{C} 5-\mathrm{C} 4-\mathrm{H} 4$ & 119.8 \\
\hline $\mathrm{O} 2-\mathrm{S} 1-\mathrm{C} 10$ & $109.57(10)$ & $\mathrm{C} 6-\mathrm{C} 5-\mathrm{C} 4$ & $120.88(19)$ \\
\hline $\mathrm{O} 3-\mathrm{S} 1-\mathrm{C} 9$ & $108.24(9)$ & $\mathrm{C} 6-\mathrm{C} 5-\mathrm{H} 5$ & 119.6 \\
\hline $\mathrm{O} 2-\mathrm{S} 1-\mathrm{C} 9$ & $105.18(9)$ & $\mathrm{C} 4-\mathrm{C} 5-\mathrm{H} 5$ & 119.6 \\
\hline $\mathrm{C} 10-\mathrm{S} 1-\mathrm{C} 9$ & $103.09(9)$ & $\mathrm{C} 5-\mathrm{C} 6-\mathrm{C} 7$ & $118.24(18)$ \\
\hline $\mathrm{C} 8-\mathrm{N} 1-\mathrm{C} 1$ & $122.54(16)$ & $\mathrm{C} 5-\mathrm{C} 6-\mathrm{H} 6$ & 120.9 \\
\hline $\mathrm{C} 8-\mathrm{N} 1-\mathrm{H} 1$ & $117(2)$ & $\mathrm{C} 7-\mathrm{C} 6-\mathrm{H} 6$ & 120.9 \\
\hline $\mathrm{C} 1-\mathrm{N} 1-\mathrm{H} 1$ & $120(2)$ & $\mathrm{C} 6-\mathrm{C} 7-\mathrm{C} 2$ & $122.18(17)$ \\
\hline $\mathrm{C} 8-\mathrm{N} 2-\mathrm{N} 3$ & $109.28(14)$ & $\mathrm{C} 6-\mathrm{C} 7-\mathrm{N} 2$ & $122.22(16)$ \\
\hline $\mathrm{C} 8-\mathrm{N} 2-\mathrm{C} 7$ & $124.09(14)$ & $\mathrm{C} 2-\mathrm{C} 7-\mathrm{N} 2$ & $115.60(15)$ \\
\hline $\mathrm{N} 3-\mathrm{N} 2-\mathrm{C} 7$ & $126.61(13)$ & $\mathrm{N} 4-\mathrm{C} 8-\mathrm{N} 2$ & $111.47(16)$ \\
\hline $\mathrm{C} 9-\mathrm{N} 3-\mathrm{N} 2$ & $100.69(13)$ & $\mathrm{N} 4-\mathrm{C} 8-\mathrm{N} 1$ & $128.56(17)$ \\
\hline $\mathrm{C} 8-\mathrm{N} 4-\mathrm{C} 9$ & $100.67(15)$ & $\mathrm{N} 2-\mathrm{C} 8-\mathrm{N} 1$ & $119.95(15)$ \\
\hline
\end{tabular}




\begin{tabular}{|c|c|c|c|}
\hline $\mathrm{O} 1-\mathrm{C} 1-\mathrm{N} 1$ & $120.47(19)$ & $\mathrm{N} 3-\mathrm{C} 9-\mathrm{N} 4$ & $117.88(15)$ \\
\hline $\mathrm{O} 1-\mathrm{C} 1-\mathrm{C} 2$ & $123.5(2)$ & $\mathrm{N} 3-\mathrm{C} 9-\mathrm{S} 1$ & $121.05(13)$ \\
\hline $\mathrm{N} 1-\mathrm{C} 1-\mathrm{C} 2$ & $116.01(16)$ & $\mathrm{N} 4-\mathrm{C} 9-\mathrm{S} 1$ & $120.94(13)$ \\
\hline $\mathrm{C} 7-\mathrm{C} 2-\mathrm{C} 3$ & $118.12(18)$ & $\mathrm{S} 1-\mathrm{C} 10-\mathrm{H} 10 \mathrm{~A}$ & 109.5 \\
\hline $\mathrm{C} 7-\mathrm{C} 2-\mathrm{C} 1$ & $121.66(16)$ & $\mathrm{S} 1-\mathrm{C} 10-\mathrm{H} 10 \mathrm{~B}$ & 109.5 \\
\hline $\mathrm{C} 3-\mathrm{C} 2-\mathrm{C} 1$ & $120.22(17)$ & $\mathrm{H} 10 \mathrm{~A}-\mathrm{C} 10-\mathrm{H} 10 \mathrm{~B}$ & 109.5 \\
\hline $\mathrm{C} 4-\mathrm{C} 3-\mathrm{C} 2$ & $120.10(18)$ & $\mathrm{S} 1-\mathrm{C} 10-\mathrm{H} 10 \mathrm{C}$ & 109.5 \\
\hline $\mathrm{C} 4-\mathrm{C} 3-\mathrm{H} 3$ & 119.9 & $\mathrm{H} 10 \mathrm{~A}-\mathrm{C} 10-\mathrm{H} 10 \mathrm{C}$ & 109.5 \\
\hline $\mathrm{C} 2-\mathrm{C} 3-\mathrm{H} 3$ & 119.9 & $\mathrm{H} 10 \mathrm{~B}-\mathrm{C} 10-\mathrm{H} 10 \mathrm{C}$ & 109.5 \\
\hline $\mathrm{C} 3-\mathrm{C} 4-\mathrm{C} 5$ & $120.47(18)$ & & \\
\hline $\mathrm{C} 8-\mathrm{N} 2-\mathrm{N} 3-\mathrm{C} 9$ & $-0.34(19)$ & $\mathrm{C} 8-\mathrm{N} 2-\mathrm{C} 7-\mathrm{C} 2$ & $0.5(2)$ \\
\hline $\mathrm{C} 7-\mathrm{N} 2-\mathrm{N} 3-\mathrm{C} 9$ & $178.37(16)$ & $\mathrm{N} 3-\mathrm{N} 2-\mathrm{C} 7-\mathrm{C} 2$ & $-178.05(17)$ \\
\hline $\mathrm{C} 8-\mathrm{N} 1-\mathrm{C} 1-\mathrm{O} 1$ & $-176.2(3)$ & $\mathrm{C} 9-\mathrm{N} 4-\mathrm{C} 8-\mathrm{N} 2$ & $0.2(2)$ \\
\hline $\mathrm{C} 8-\mathrm{N} 1-\mathrm{C} 1-\mathrm{C} 2$ & $3.1(3)$ & $\mathrm{C} 9-\mathrm{N} 4-\mathrm{C} 8-\mathrm{N} 1$ & $178.8(2)$ \\
\hline $\mathrm{O} 1-\mathrm{C} 1-\mathrm{C} 2-\mathrm{C} 7$ & $179.4(3)$ & $\mathrm{N} 3-\mathrm{N} 2-\mathrm{C} 8-\mathrm{N} 4$ & $0.1(2)$ \\
\hline $\mathrm{N} 1-\mathrm{C} 1-\mathrm{C} 2-\mathrm{C} 7$ & $0.1(3)$ & $\mathrm{C} 7-\mathrm{N} 2-\mathrm{C} 8-\mathrm{N} 4$ & $-178.68(16)$ \\
\hline $\mathrm{O} 1-\mathrm{C} 1-\mathrm{C} 2-\mathrm{C} 3$ & $-0.6(4)$ & $\mathrm{N} 3-\mathrm{N} 2-\mathrm{C} 8-\mathrm{N} 1$ & $-178.63(19)$ \\
\hline $\mathrm{N} 1-\mathrm{C} 1-\mathrm{C} 2-\mathrm{C} 3$ & $-179.9(2)$ & $\mathrm{C} 7-\mathrm{N} 2-\mathrm{C} 8-\mathrm{N} 1$ & $2.6(3)$ \\
\hline $\mathrm{C} 7-\mathrm{C} 2-\mathrm{C} 3-\mathrm{C} 4$ & $0.4(3)$ & $\mathrm{C} 1-\mathrm{N} 1-\mathrm{C} 8-\mathrm{N} 4$ & $177.0(2)$ \\
\hline $\mathrm{C} 1-\mathrm{C} 2-\mathrm{C} 3-\mathrm{C} 4$ & $-179.7(2)$ & $\mathrm{C} 1-\mathrm{N} 1-\mathrm{C} 8-\mathrm{N} 2$ & $-4.5(3)$ \\
\hline $\mathrm{C} 2-\mathrm{C} 3-\mathrm{C} 4-\mathrm{C} 5$ & $0.4(3)$ & $\mathrm{N} 2-\mathrm{N} 3-\mathrm{C} 9-\mathrm{N} 4$ & $0.5(2)$ \\
\hline $\mathrm{C} 3-\mathrm{C} 4-\mathrm{C} 5-\mathrm{C} 6$ & $-0.1(3)$ & $\mathrm{N} 2-\mathrm{N} 3-\mathrm{C} 9-\mathrm{S} 1$ & $-175.38(13)$ \\
\hline $\mathrm{C} 4-\mathrm{C} 5-\mathrm{C} 6-\mathrm{C} 7$ & $-1.0(3)$ & $\mathrm{C} 8-\mathrm{N} 4-\mathrm{C} 9-\mathrm{N} 3$ & $-0.5(2)$ \\
\hline $\mathrm{C} 5-\mathrm{C} 6-\mathrm{C} 7-\mathrm{C} 2$ & $1.8(3)$ & $\mathrm{C} 8-\mathrm{N} 4-\mathrm{C} 9-\mathrm{S} 1$ & $175.42(14)$ \\
\hline $\mathrm{C} 5-\mathrm{C} 6-\mathrm{C} 7-\mathrm{N} 2$ & $-177.84(18)$ & $\mathrm{O} 3-\mathrm{S} 1-\mathrm{C} 9-\mathrm{N} 3$ & $-34.09(18)$ \\
\hline $\mathrm{C} 3-\mathrm{C} 2-\mathrm{C} 7-\mathrm{C} 6$ & $-1.5(3)$ & $\mathrm{O} 2-\mathrm{S} 1-\mathrm{C} 9-\mathrm{N} 3$ & $-163.43(15)$ \\
\hline $\mathrm{C} 1-\mathrm{C} 2-\mathrm{C} 7-\mathrm{C} 6$ & $178.53(19)$ & $\mathrm{C} 10-\mathrm{S} 1-\mathrm{C} 9-\mathrm{N} 3$ & $81.78(17)$ \\
\hline $\mathrm{C} 3-\mathrm{C} 2-\mathrm{C} 7-\mathrm{N} 2$ & $178.19(17)$ & $\mathrm{O} 3-\mathrm{S} 1-\mathrm{C} 9-\mathrm{N} 4$ & $150.12(17)$ \\
\hline $\mathrm{C} 1-\mathrm{C} 2-\mathrm{C} 7-\mathrm{N} 2$ & $-1.8(3)$ & $\mathrm{O} 2-\mathrm{S} 1-\mathrm{C} 9-\mathrm{N} 4$ & $20.79(18)$ \\
\hline $\mathrm{C} 8-\mathrm{N} 2-\mathrm{C} 7-\mathrm{C} 6$ & $-179.84(18)$ & $\mathrm{C} 10-\mathrm{S} 1-\mathrm{C} 9-\mathrm{N} 4$ & $-94.00(18)$ \\
\hline $\mathrm{N} 3-\mathrm{N} 2-\mathrm{C} 7-\mathrm{C} 6$ & $1.6(3)$ & & \\
\hline
\end{tabular}

Hydrogen-bond geometry $\left(\AA,{ }^{\circ}\right)$

\begin{tabular}{lllll}
\hline$D-\mathrm{H} \cdots A$ & $D-\mathrm{H}$ & $\mathrm{H} \cdots A$ & $D \cdots A$ & $D-\mathrm{H} \cdots A$ \\
\hline $\mathrm{N} 1-\mathrm{H} 1 \cdots \mathrm{O} 2^{\mathrm{i}}$ & $0.88(1)$ & $2.23(1)$ & $3.072(2)$ & $160(3)$ \\
\hline
\end{tabular}

Symmetry code: (i) $-x+1, y+1 / 2,-z$. 